\title{
Getting in Touch with the Lost Self: Vicarious and
}

\section{Affective Touch in Depersonalisation}

3

\author{
Anna Ciaunica*1,2,3, Jyothisa Mathew ${ }^{4}$, Ophelia Deroy ${ }^{5,6,7}$, Merle Fairhurst ${ }^{4,5,6}$ \\ Affiliations: \\ 1Centre for Philosophy of Science, University of Lisbon, Campo Grande, 1749-016 Lisbon, \\ Portugal \\ 2 Institute of Philosophy, University of Porto, via Panoramica s/n 4150-564, Porto Portugal \\ 3 Institute of Cognitive Neuroscience, University College London, WC1N 3AR, London, UK \\ 4 Institute for Psychology, Bundeswehr University Munich, Munich, Germany \\ 5Faculty of Philosophy \& Philosophy of Science, Ludwig Maximilian University, Munich, \\ Germany \\ 6 Munich Center for Neuroscience, Ludwig Maximilian University, Munich, Germany \\ 7 School of Advanced Study, University of London, London, UK.

\begin{abstract}
*Corresponding author: Dr Anna Ciaunica : a.ciaunica@ucl.ac.uk Centre for Philosophy of Science, Faculty of Science, University of Lisbon, Campo Grande, 1749-016 Lisbon, Portugal
\end{abstract}

\begin{abstract}
We conducted an online study featuring two experiments in order to examine the relationship between depersonalization experiences (DP) (i.e. feelings of being detached from one's self and body) and vicarious affective touch and self-touch. Experiment 1 examined to what extent DP traits modulate the perceived pleasantness and/or vividness of tactile experiences as imagined being received by the self and other. In experiment 2 we designed a new affective self-touch intervention in order to explore the effect of CT-optimal self-touch stroking on one's dorsal forearm on the perceived pleasantness and vividness of tactile experiences as being received by the self and other. We found that low DP individuals reported higher perceived pleasantness and vividness rating for touch. By contrast, the high DP cohort rated all touch experiences as significantly less pleasant. No significant interaction effects for vividness ratings of touch experiences across low and high DP. In addition, our results suggest that people with low DP rate the perceived pleasantness of the imagined social touch experiences as received by the self higher than if received by the other. Interestingly, in high DP individuals, there is no difference in the perceived pleasantness of affective touch imagined as being received by the self vs the other. Finally, we found that both low and high DP participants, following our tailored CT-optimal affective self-touch intervention on one's own body, report significantly higher ratings of vividness of tactile perception.
\end{abstract}

Keywords : affective touch, vicarious touch, self-touch, sense of self, depersonalization

\section{Author contributions}

$\mathrm{AC}$ and MF designed the study. JM and MF collected and analyzed the data. AC secured the funding and wrote the first draft of the manuscript. JM and MF wrote the Methods and Results sections. AC, JM, OD, MF revised the final version of the manuscript. 
The sense of self, the subjective feeling of being an 'I' or a 'self' bound to my body as distinct from the world and others, is a fundamental aspect of our everyday experiences (Gallagher 2000; Damasio 2000; Zahavi 2005; Blanke \& Metzinger 2009; see Quin et al. 2020 for a review).

Depersonalisation (DP) is a common dissociative experience characterized by distressing feelings of being detached or estranged from one's self and body. It can also combine with similar feelings of detachment from one's surroundings (DR Derealisation) (Sierra \& Berrios 1997). As a person with DP explains: “when I'm having an episode of depersonalisation, it feels more like I'm watching myself doing things, but I'm not present for it. I'm witnessing myself... I 'know' I'm in control, but I'm not 'feeling' in control”' (Perkins 2021:44). This self-detachment induces uncanny sensations of not being fully present or real, and of losing touch with one's self, body and the world (Simeon \& Abugel, 2006; Sierra 2009; Ciaunica \& Charlton, 2018).

DP is often triggered by traumatic life events, substance use, severe stress, prolonged depression, extreme fatigue (van Heugten et al. 2015; Titubos et al. 2018). DP has a prevalence of around $1-2 \%$ of the population, with an onset in adolescence or early adulthood (Baker et al. 2003; Hunter et al. 2003,2004; Michal et al. 2007; see Salami et al. 2020 for a recent review). Chronic states of depersonalisation and derealisation symptoms may lead to a diagnosis of Depersonalisation-Derealisation Disorder (DDD) (American Psychiatric Association 2013). The classification of DP and DDD as dissociative conditions is subject to a nuanced approach. Millman and colleagues (2021) recently used a latent profile analysis to examine symptom variability in clinical depersonalisation. They analysis identified two qualitatively different categories of dissociative symptoms: "compartmentalization" and “detachment "(Brown, 2006; Holmes et al. 2005). Detachment is defined as the subjective sense of separation typically encountered in DP, whereas compartmentalization refers to a dissociative inability to have deliberate control over actions. In this paper we are concerned with transient and mild experiences of DP as self-detachment. 
The experience of a disconnect between the self and the body - strikingly described as feeling trapped in one's head (mind) and outside one's body (Ciaunica et al. 2020) — is one of the most frequently cited symptoms in DP (Sierra \& David, 2011). Sierra (2009) lists four prominent types of anomalous body experiences in DP: (1) lack of body ownership (2) feelings of loss of agency (3) disembodiment feelings and (4) somatosensory distortions. Empirical support for this disrupted bodily sensory processing comes from studies that demonstrate disrupted physiological responses in patients with DDD, compared to healthy participants (Dewe et al., 2018; Owens et al., 2015; Sierra et al., 2002). DP has also been linked to disrupted activity in neuronal regions underlying somatic processing (Lemche, et al., 2013; Medford et al., 2016) and the vestibular system (Jáuregui Renaud, 2015), which is responsible for providing information about the body's position in space (Ferrè \& Haggard, 2016).

Importantly, people experiencing DP report a significant impact of this self-detachment on the quality of their social and emotional life, leading to social isolation: "Feeling unreal and disconnected from my body and the world around me caused me to lose interest in the people and hobbies I used to love" (Perkins 2021: 190).

Recent work has emphasised the multisensory and embodied basis of the human sense of self and self-consciousness as it unfolds dynamically throughout the lifespan (Alais et al. 2010; Deroy et al. 2014; Faivre et al. 2017; Tsakiris 2017; Ciaunica et al. 2021a,b). Among the senses, tactile perception and particularly self-touch has recently received close attention (Gallace \& Spence 2010; Ratcliffe 2013, Ciaunica \& Fotopoulou 2017; Boehme \& Olausson 2021). The key idea is that tactile perception is inherently relational and reciprocal: the 'toucher' and the 'touched' need to be physically proximal, to 'share' the experience of touch (passive or active) (Merleau-Ponty 1962).

While traditionally self-awareness is considered to emerge through visuospatial and distal mirror self-recognition (Lewis et al. 1989), recent work outlined that humans first perceptually 'meet' other people's bodies (via proximal skin-to-skin interactions) (Ciaunica 2017). Indeed, touch is one of the first of our senses to develop in the human embryo (Gottlieb 1976), and provides us thereby with our most basic and earliest means of perceiving both the self and the social and physical environment, already in utero (Ciaunica et al. 2021a,d). Hence it has been hypothesised that touch perception might represent a fundamental 
step in the development of both self- and other-awareness, as well as self-other distinction (Rochat \& Striano 2000; McGlone et al. 2014; Ciaunica \& Fotopoulou, 2017; Ciaunica \& Crucianelli 2019). People are also more likely turn to touch for subjective reassurance, when their sense of reality is shaken (Fairhurst et al 2018; Fairhurst \& Deroy 2019).

The pivotal role of touch for the bodily self has received further attention especially after the discovery that gentle, caress-like stroking on the hairy skin at an optimal velocity $(3-10 \mathrm{~m} / \mathrm{s}$, Löken et al., 2009) and skin temperature (Ackerley et al. 2014) activates a specific subclass of receptors. The so-called C-tactile (CT) afferents in human hairy skin code for pleasant touch, (Johansson et al. 1988; Nordin 1990; Vallbo et al 1993) and activates the neural level the posterior insula and orbitofrontal cortices (McGlone et al. 2012; Trotter et al. 2016).

Previous work outlined the beneficial effects of positive touch experiences produced by CT optimal stroking touch (Fairhurst et al. 2014; Liljencrantz et al. 2017; Pawling et al. 2017; see McGlone et al. 2007; Morrison et al. 2010 for reviews).The so-called affective or social touch is considered to subserve key mechanisms of social bonding, reducing feelings of social exclusion (von Mohr et al., 2017), soothing pain (Krahe et al., 2016; von Mohr et al., 2018) and communicating social support (Kirsch et al., 2018; Fotopoulou et al. 2021). Evidence for the rewarding nature of affective touch comes also from studies showing increased activity of mu-opioid receptors (MOR) - considered to underpin the maintenance of close social connectedness in humans (Pearce et al. 2017) - during social touch (Nummenmaa et al. 2016)

Interestingly, several studies found a strong link between the bodily self and the mechanisms underlying vicarious touch, i.e. the automatic simulation of touch observed on another person's body (Keysers et al. 2004; Adler \& Gillmeister 2019). Neuroimaging work outlined that the passive observation of touch experienced by another individual elicits vicarious activity in similar brain regions as those activated when touch is received by the individual on her own body (Keysers et al. 2010; Lamm et al. 2011). Significant individual variability has been reported in exploring vicarious responses to others' sensory experiences (Gillmeister et al. 2017).

For example, Morrison and colleagues (2011) reported that vicarious responses to dynamic stroking touch are velocity tuned and socially specific. More recent studies found that vicarious ratings of affective touch match the velocity tunning of CT afferents in adults 
155 (Walker et al. 2017) and in children aged between 8 and 11 (Haggarty et al. 2021). In these studies, participants viewed and rated short videos depicting one individual being touched by another individual at different skin sites (back, upper arm, ventral forearm, dorsal forearm and palm) at 3 different velocities (static, $3 \mathrm{~cm} / \mathrm{s}, 30 \mathrm{~cm} / \mathrm{s}$ ). The authors report that the highest ratings of reported pleasantness were for observing gentle stroking touch delivered at CT optimal stroking.

Although self-touch and affective touch are increasingly recognized as playing a key role in establishing one's sense of self and social connectedness, little is known about the relationship between affective touch and DP. This gap is surprising given that numerous selfreports from people experiencing depersonalisation point to the importance of maintaining close tactile contact and social interactions with the physical and social environment in order to enhance one's sense of self. In the words of one interviewees: "When the depersonalisation is very deep, (...) it feels like that constant source of interaction is the only thing that allows me to maintain a connection with the world. I'll also seek physical contact with whoever I'm with.” (Ciaunica \& Charlton 2018). Or again, coming from a person with lived experiences of DP : "I really like when people scratch me or twist my arms or just touch me. It puts me back in my body and makes me feel cared for" (Perkins 2021:87).

Here, we conducted an online study featuring two experiments aiming to examine the relationship between DP experiences and vicarious affective touch and self-touch. The first experiment built upon previous work by Walker and colleagues (2017) and explored to what extent DP traits modulate the perceived pleasantness and/or vividness of tactile experiences as imagined being received by the self and other. We hypothesised that the higher experiences of depersonalisation (as measured by the Cambridge Depersonalisation Scale (CDS-29), Sierra $\&$ Berrios 2000, see Methods below), the lower the perceived pleasantness and/or vividness of the vicarious tactile experiences as imagined being received by both the self and other. By contrast, people with low occurrences of depersonalisation should report higher perceived pleasantness and vividness of tactile experiences imagined as being received by the self, as opposed to the other.

In the second experiment we designed a new affective self-touch task (adapted from Walker et al. 2017), aiming to explore the effect of CT-optimal self-touch stroking on one's dorsal forearm on the perceived pleasantness and vividness of tactile experiences as being received by the self and other. We hypothesised that the dynamic and affective self-touch stroking (as 
opposed to passive self-touch) will enhance the perceived vividness and pleasantness of the social touch as imagined being received by the self and other in both people with high and low DP experiences.

\section{Materials and Methods}

197

198

199

200

201

202

203

204

205

206

\subsection{Participants}

409 participants were pre-screened via the online platform SoSci Survey (Leiner, 2016) using the Cambridge Depersonalization Scale (CDS-29) (Sierra \& Berrios, 2000). From these, 108 eligible participants (Male $=38$, Female $=67$, non-binary $=2$, prefer not to say $=1)$ (mean age $=$ 26.92, $\mathrm{SD}=5.034$ ) completed the study in two groups: (a) 57 participants in the High DP group with their score $\geq 50$; and (b) and 51participants in the Low DP group with their scores $\leq 20$ on the CDS-29. CDS-29 score calculation used a procedure developed by Kanayama et al. (2009) which ensures that the DP scores of the two groups are meaningfully distinct. Previous studies have demonstrated that this procedure should yield a sensitivity threshold of 75.5\% for DP patients in the High DP group (Sierra et al., 2005). Other criteria included: (a) between 18 to 40 years old, (b) fluent English speakers, (c) right-handed and (d) without a history of neurological illness. Informed consent was obtained from all participants before the start of the experiment according to procedures approved by the Ethics Committee of the Universität der Bundeswehr München. The experiment was conducted in accordance with the Declaration of Helsinki.

\subsection{Questionnaires}

Cambridge Depersonalization Scale (CDS-29 henceforth) (Sierra \& Berrios, 2000) is a 29 items standard questionnaire used to evaluate the severity of occurrence of depersonalization experiences. CDS-29 asks the participants to estimate the frequency and duration of their DP symptoms. Frequency is characterized as how often the symptoms have occurred in the last 6 months and is measured on a 5-point Likert rating scale ranging from 0 (never) to 4 (all the time) for each of the 29 items. Duration is measured on a 7-point Likert rating scale ranging from 0 (few seconds) to 6 (more than a week) for each of the 29 items. A total score is calculated by adding up the total frequency and duration scores and it can range between 0 to 
290. CDS-29 has good statistical properties (Aponte-Soto, Vélez-Pastrana, Martínez-Taboas, \& González, 2014; Fagioli et al., 2015; Migliorini et al., 2012; Sierra \& Berrios, 2000; Sugiura et al., 2009) with internal reliability for different language versions reported between 0.89-0.94 (Cronbach alpha). Moreover, previous research has extracted four subscales from CDS-29 (Sierra, Baker, Medford, \& David, 2005): Anomalous Body Experience, Emotional Numbing, Anomalous Subjective Recall, and Alienation from Surroundings.

Social Touch Questionnaire (STQ henceforth) (Wilhelm et al., 2001) is a 20-item assessing the participants' attitude towards social situations involving touch. It consists of items that measure (i) giving/providing and (ii) getting/receiving touch along with touch involving strangers and acquaintances. The items are measured on a 5-point Likert rating scale ranging from 0 (not at all) to 4 (extremely). An STQ score is calculated by adding up the values for each item after carefully ensuring that half of the items are negatively scored. (Negative items: $2,3,5,7,8,10,13,16,17,19)$. In this study, additionally a 'Get' and 'Give' score was calculated. The former score indicates how comfortable a participant is being touched (getting or receiving a touch), whereas the latter score indicates how comfortable the participant is and their attitudes towards giving touch to friends or acquaintances. The 'Give' (Give items: $4,5,6,9,10,13,14,15,20)$ and 'Get' score (Get items: 1,2,3,7,8,11,12,17,18,19) were calculated as a sum of their scores for their respective items.

Longing for interpersonal touch picture questionnaire (LITPQ henceforth) is a rather novel method to measure longing for touch. It consists of two components i.e. touch frequency and touch wish. Touch frequency is calculated by asking participants how often they had performed a specific type of touch in the past week whereas touch wish is calculated by asking participants how often they would have wanted to experience a specific type of touch in the past week. In the present study, to measure both these components, participants were required to respond on a slider under each picture ranging from 0 to 100 with 50 as the midpoint. For this study, pictures with four different types of touch such as holding, stroking, kissing and hugging were selected. Three scores were calculated as a result of this questionnaire namely: (i) touch wish, (ii) touch frequency and (iii) total LITPQ score. Touch frequency and touch wish was calculated as an average across all touch types. LITPQ score was calculated by dividing touch wish by touch frequency, thereby creating a ratio of the two scores.

\subsection{General Procedure}

Initial pre-screening for depersonalisation traits via the CDS-29, was followed by a short 
experiment 1, participants were asked to imagine a social touch event occurring either to themselves or another person (SELF/OTHER). In experiment 2, participants were again asked to imagine being touched but this time, before and after a self-touch intervention that we have designed (SELF TOUCH INTERVENTION). These two experiments are described in detail below. The order of the two experiments was randomised across participants. Having completed the two experiments, all participants filled (a) the Social Touch Questionnaire (STQ) and (b) the Longing for Touch Interpersonal Picture Questionnaire (LITPQ).

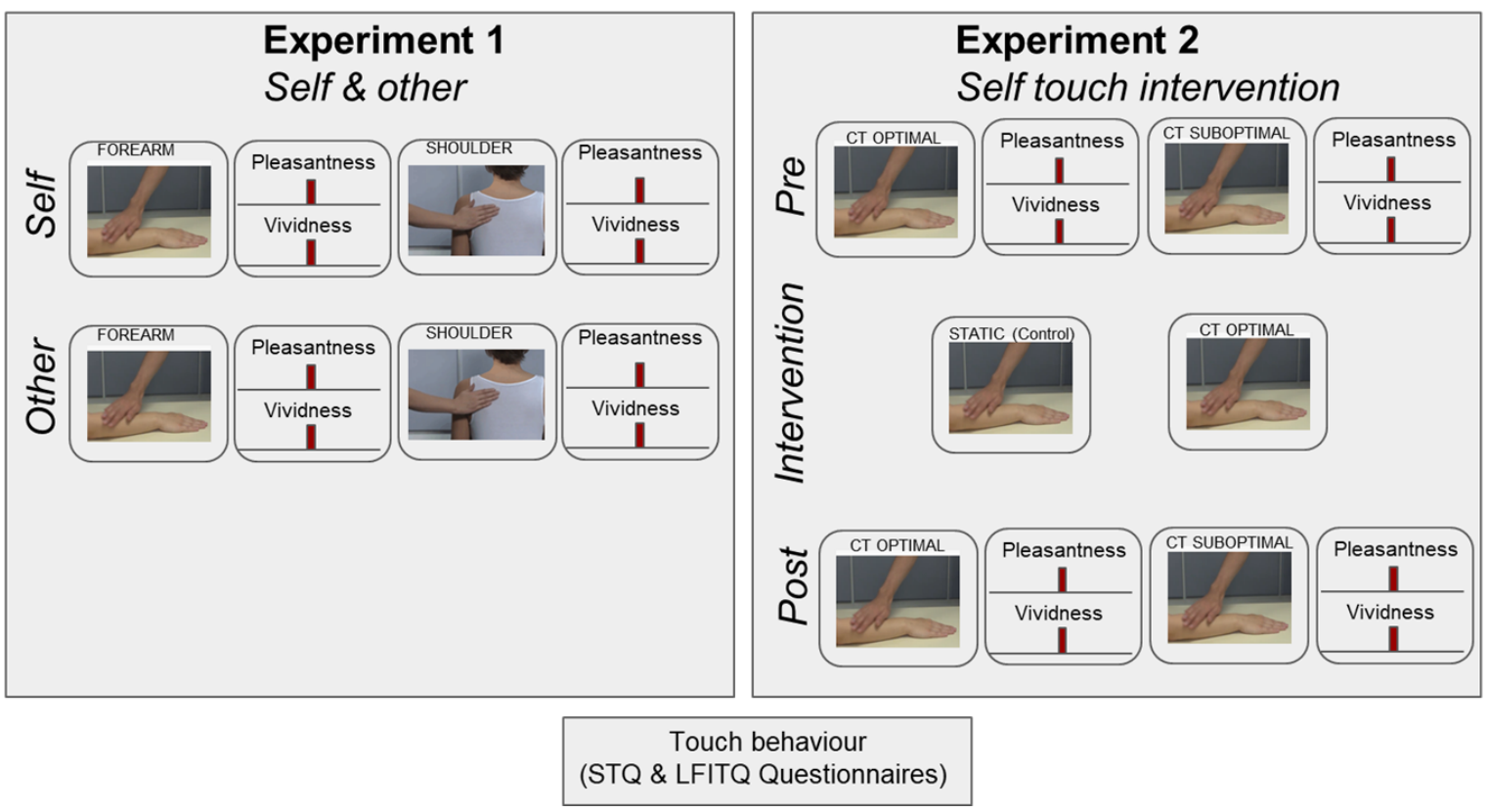

Fig 1: Overview of the Experimental Design and Procedure

\subsection{Design and Procedure Experiment 1 SELF/OTHER}

273 In experiment 1, participants viewed a series of short videos (6s) depicting an individual's

274 hand stroking another individual's dorsal forearm at CT-optimal velocity (see Fig 1). In the videos presented, the gender of the person being touched were matched according to the selfdeclared gender of the participant; in all cases the person performing the stroking was female.

277 Previous work suggested that both sexes are likely to respond more homogenously to female 278 stroking (Gazzola et al., 2012). We first manipulated the "WHERE" factor, by presenting 279 videos in which participants observed a person gently stroking either another person's (i) 280 dorsal forearm (the FOREARM condition) or (ii) back (the BACK condition) at the CT281 optimal speed. Secondly, we manipulated the "WHO" factor, namely: (i) in half of the trials, 
participants were asked to imagine these tactile experiences as if it were happening to themselves (the SELF condition). (ii) While in the other half, they were asked to imagine these as happening to someone else (the OTHER condition). In this experiment, the participants were presented with 1 practise block containing 1 trial followed by 2 test blocks containing 5 trials each for the 'self' and 'other' blocks. Participants then completed the two questionnaires STQ and LITPQ.

The study thus used a $2 \times 2 \times 2$ within-subjects design with three independent variables: (a) Experimental GROUP (Trait Depersonalisation - Low DP vs. High DP), (b) WHO was the imagined person receiving the affective touch (SELF vs. OTHER); (c) WHERE, i.e., the place on the body where the touch was delivered in the videos we have created with two levels (BACK vs. dorsal FOREARM). The dependent variables of this experiment were perceived pleasantness and vividness as rated on visual analogue scales presented after each video.

\subsection{Design and procedure Experiment 2 INTERVENTION}

In experiment 2, participants viewed short videos (6s) showing an individual's hand stroking another individual's dorsal forearm (see Fig. 1). Here we have manipulated how the stroking was performed ("HOW" factor) with videos showing stroking at either CT optimal velocity (3 $\mathrm{m} / \mathrm{s}$ ) or CT suboptimal velocity $(30 \mathrm{~m} / \mathrm{s})$. Half of the participants (INTERVENTION group) were shown a video with an incorporated audiovisual metronome, giving them the CToptimal pace of affective touch stroking. They were instructed to copy and perform on their own left dorsal forearm the self-touch stroking with the CT-optimal velocity as shown in the video. In the CONTROL group, participants were instructed to rest their right hand passively on the opposite arm for the same duration as the self-intervention touch (i.e. 6 seconds). The rationale behind this condition was to control for the effect of dynamic affective self-touch as opposed to passive self-touch. The structure of this intervention experiment followed a prepost design with participants completing an imagined touch block before a touch intervention (PRE), followed by a second imagined touch block (POST). The training session consisted of one video followed by the two rating scales. PRE and POST blocks consisted of five trials each. The intervention condition consisted of two trials. 
VELOCITY of the video stimuli presented with two levels (CT-optimal vs. non-CT-optimal).

318 The dependent variables of this experiment were perceived pleasantness and vividness which were calculated as delta scores by subtracting the pre-test scores from the post-test scores.

\subsection{Data types and analysis}

The three questionnaires used in the study for pre-screening and the main study were subjective self-report questionnaires. While CDS and STQ included Likert-rating scales providing ratio data, participants had to respond to LITPQ items on a slider response scale for each picture also providing ratio data. In both experiments, the variables of perceived vividness and pleasantness were calculated using self-report measures on a slider response scale and reaction time was recorded additionally, both, providing ratio data. The prescreening survey was conducted using the SosciSurvey (Leiner, 2016) platform. The experiment created and hosted using the Gorilla Experiment builder (www. gorilla.sc; AnwylIrvine, Massonnié, Flitton, Kirkham \& Evershed, 2018). Data was organised using Microsoft Excel (Microsoft Corporation, 2018) and analysed using RStudio (RStudio Team, 2020). Tests conducted include student t-tests, repeated measures ANOVAs and multiple regression.

\section{Results}

\subsection{Results STQ Touch behaviour}

341 Using a student t-test, we found a significant effect of experimental GROUP on reports of 342 touch behaviour as captured by the STQ $(\mathrm{t}(106)=-3.22, p<0.01)$, with significantly higher total scores for low DP individuals $(\mathrm{M}=46.15)$ than high DP individuals $(\mathrm{M}=38.28)$.

344 Additionally, we compared 'give 'vs. 'get' items (that is instances in which the individual is either touching or being touched). We found that high DP $(M=19.19)$ individuals rate "get"

346 (being touched) items significantly lower $(\mathrm{t}(106)=-4.23, p<0.001)$ compared to low DP

$347(\mathrm{M}=24.72)$ individuals. This is not the case for "give" (touching) items $(\mathrm{t}(106)=-1.47, p=$ 348 0.14) (Fig 2). 


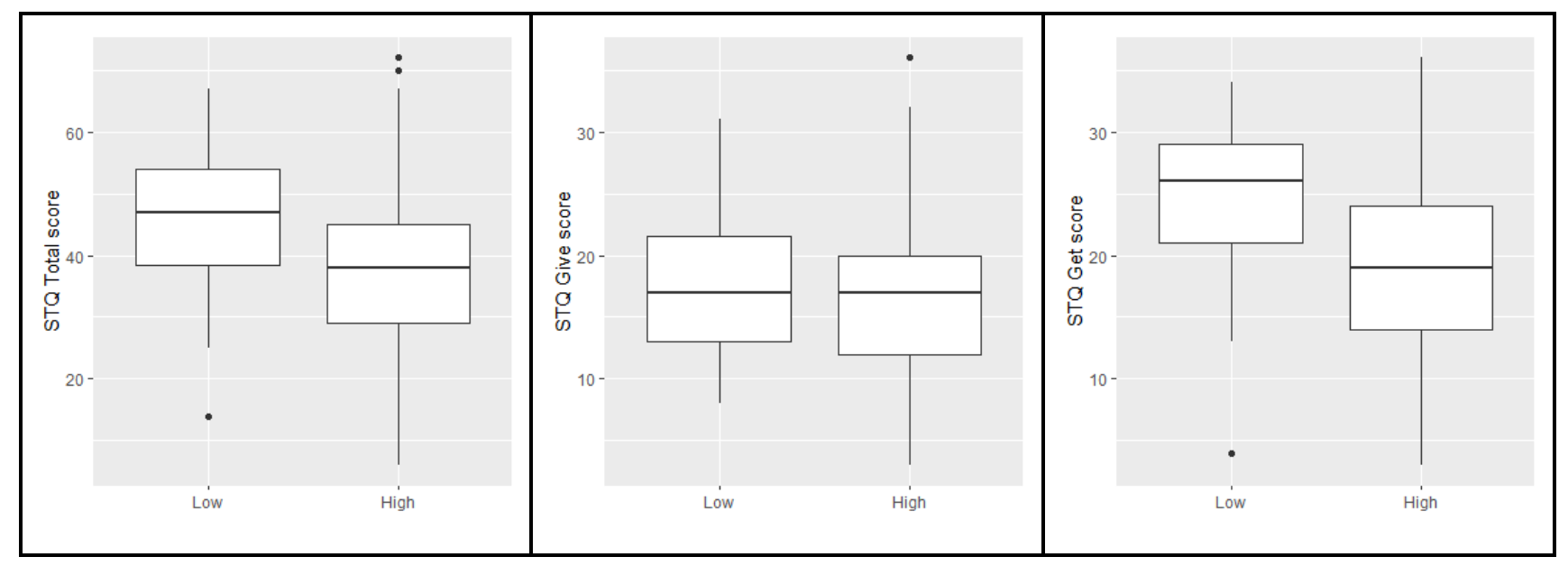

Figure 2: Experiment 1 - Touch behaviours in low and high DP individuals measured using the Social Touch

Questionnaire

\subsection{Results Experiment 1}

Using a 2X2X2 ANOVA with factors: (i) WHO (who was imagined as receiving the touch: self vs other), (ii) WHERE (where the touch was delivered: back vs dorsal forearm), and (iii) our experimental GROUP (low vs. high DP), we found several significant effects. Firstly, for the perceived pleasantness variable, we find a significant interaction between our GROUP $x$ WHO factors $(\mathrm{F}=13.38195, p<0.001)$. Namely: the low DP group rated imagined tactile experiences personally experienced (SELF) as more pleasant than imagined experiences as experienced by the other (OTHER). By contrast, no difference between "self" and "other" conditions are observed for the high DP group (see Fig 3).

Additionally, we find a significant main effect for pleasantness for (i) the experimental

$366 \operatorname{GROUP}(\mathrm{F}=15.85186, p<0.001)$ and (ii) WHERE $(\mathrm{F}=5.911614, p<0.001)$ touch was

367 imagined ('FOREARM' vs 'BACK'), confirming previous reports with the imagined "back"

368 touch scenarios being rated significantly more pleasant than the dorsal forearm. No

369 significant interaction effects were found for vividness ratings (though we see a main effect of WHO $(\mathrm{F}=7.192446, p<0.001))$. 


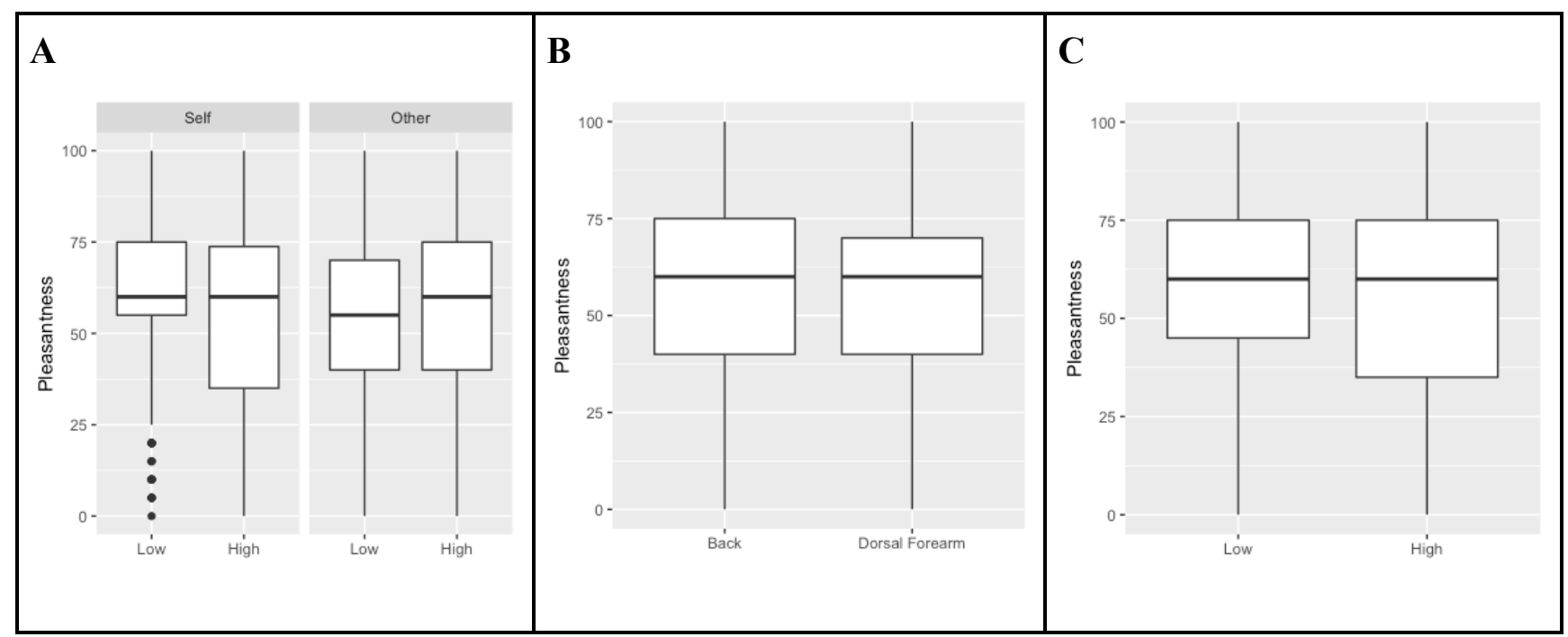

Figure 3. Experiment 1 (A) interaction between GROUP x WHO factors; (B) main effect of WHERE and (C)

\subsection{Results Experiment 2}

Using a multiple regression, including the factors (i) INTERVENTION, (ii) GROUP and (iii) CT stroking VELOCITY in our model, we identify a significant effect of intervention ( $\beta=$ 9.66, $\mathrm{SE}=3.46, t=2.78, p<0.05)$. This indicates that both low and high DP participants, following a self-touch intervention, report higher ratings of vividness of imagined tactile experiences (see Fig. 4). No significant effect on the pleasantness of the imagined tactile experiences has been found across condition.

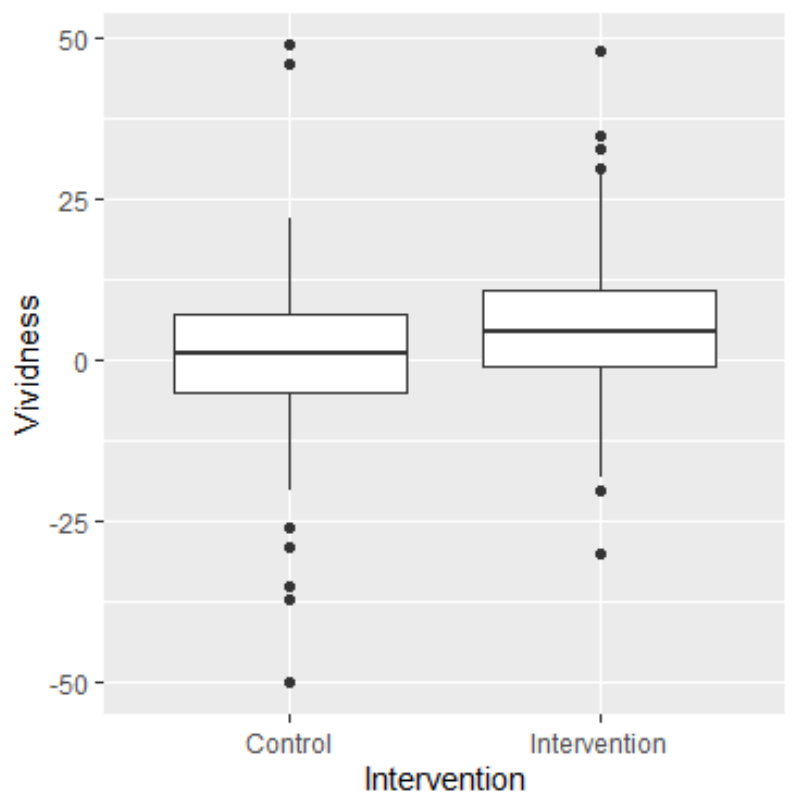

Figure 4: Results experiment 2 - Self-touch intervention and perceived vividness of imagined touch. 


\section{Discussion}

This study explored the relationship between depersonalisation experiences (feelings of being detached from one's self and body) and affective, social touch. Our analyses yielded evidence for several notable findings.

First, as predicted, we found that DP significantly modulates the subjective rating of touch experiences overall, with low DP individuals reporting higher perceived pleasantness and vividness rating for touch. By contrast, the high DP cohort rated all touch experiences as significantly less pleasant than their low DP counterparts. This suggest that people with depersonalisation benefit less from the social and affective component of tactile experiences, consistent of their common complaints of feeling affectively detached from the others and unable to 'feel love' (Simeon and Abugel 2006; Sierra 2009; Perkins 2021).

Interestingly, no significant interaction effects for vividness ratings of touch experiences across the groups of low versus high depersonalisation traits has been found. This is surprising at first glance because, in line with previous work highlighting a close link between atypical bodily self-awareness, social isolation and depersonalisation (Simeon \& Abugel 2006; Sierra \& David 2011), we have initially predicted that both the perceived pleasantness and vividness of the tactile experiences will be overall higher in for the Low DP group, and lower for the High DP group. However, our results suggest that the vividness and pleasantness features of tactile experiences are underlined by two different mechanisms in relation to DP. As we will see shortly below, this hypothesis seems to receive further support from the results of our experiment 2, where we find this time a significant effect on vividness but not pleasantness following the self-touch intervention in both low and high DP groups.

A third notable finding is that people with low DP rate the perceived pleasantness of the imagined social touch experiences as received by the self higher than those received by the other. Interestingly, in high DP individuals, there is no difference in the perceived pleasantness of affective touch imagined as being received by the self and by the other. 
420 Finally, we found that both low and high DP participants, following our tailored CT-optimal

421 affective self-touch intervention on one's own body, report significantly higher ratings of

422 vividness of tactile perception. This is an important finding because it suggests that dynamic

423 and affective self-touch stroking on one's own body may enhance the vividness of one's

424 perceptual experiences, and potentially reduce thereby feelings of disembodiment and

425 unreality in people with DP. These findings seem to be supported further by DP

426 experiencers' anecdotical self-reports highlighting the positive effect of dynamic proximal

427 tactile interactions with close others and the environment on one's sense of self (Ciaunica et

428 al. 2021; Perkins 2021). As mentioned above, interestingly, no effect of the CT-optimal self-

429 touch intervention effect has been found on the pleasantness rating of touch experiences in

430 both low and high DP groups.

431

432 In what follows we discuss in detail the interpretations and potential implications of our

433 findings in turn.

434

435 We start by noting that in our study, people with low occurrences of DP experiences score 436 significantly higher on the Social Touch Questionnaire than their high DP counterparts.

437 Again, this is in line with previous self-reports from people experiencing DP noting a

438 significant impact of their disembodiment and self-detachment on the quality of their social 439 and emotional life: "I feel like a robot, I am looking at myselffrom the outside, but it is not 440 another voice or body, it is mine, it is me, it just doesn't feel like it. Nothing makes it better 441 but being with other people makes it worse" (Baker et al., 2003: 431). Importantly, a recent 442 study by Millman and colleagues (2021) found that people clinically diagnosed with severe 443 depersonalisation tend to attribute their symptoms to social factors, which can lend insights 444 into people's subjective appraisal of their symptoms.

447 Indeed, a core feature of DP is impaired processing of emotions (Blevins et al., 2013; Sierra et 448 al.,2002b), with a persistent decrease or loss of emotional reactivity, and emotions seem to 449 lack 'realness' and 'colour' (Baker et al. 2003 ; Simeon 2004; Simeon and Abugel 2006).

450 These subjective reports seem to be supported by empirical work suggesting that DdD 451 patients show attenuated responses to emotional stimuli ( Sierra et al. 2002; Medford et al. 452 2006). Moreover, DDD patients reporting high levels of subjective anxiety show attenuation 453 of autonomic sympathetic responses (Sierra et al. 2002; 2006). 
Interestingly, as mentioned above, our study found no significant interaction effects for vividness ratings of touch experiences across the groups of low versus high depersonalisation traits. The lack of vividness effect may be explained by the fact that our study and questionnaires were conducted online. Yet, the vividness of one's experiences is closely related with one's dynamic presence in the tri-dimensional embodied reality of the here and now (as opposed to the sedentary stance of the individual facing a two-dimensional, digital reality presented on the screen). For example, a recent study (Ciaunica et al. 2021c) found positive correlation between higher depersonalisation experiences and increased digital media sedentary activities (e.g. playing computer game, watching TV, online e-meetings participation, etc). This may explain why people with low occurrences of DP report the same rates of vividness for touch experiences as their high DP counterparts. Further work is needed however to test this hypothesis in a systematic way.

Another interesting effect revealed by our first experiment is that low DP people report higher perceived pleasantness of the imagined social touch experiences as received by the self vs the other. This seems to be in line with recent trends in mind and brain research stipulating that our perceptions, cognitions and actions are intrinsically geared towards self-preservation (Panksepp, 1998; Northoff \& Panksepp, 2008; Ciaunica \& Fotopoulou 2017; Seth \& Tsakiris, 2018; Limanowski \& Friston 2018). By maintaining and regulating the physiological needs and integrity of the organism (the human body), perceptual awareness at the most basic level is inherently "selfish", that is self-centred, concerned with self-survival and self-care (Friston 2010; Seth \& Tsakiris, 2018; Ciaunica \& Crucianelli, 2019).

This idea seems to be captured nicely by the so-called 'self-prioritization or 'self-bias' effect, which is an effect demonstrating that people are faster and more accurate when processing information related to the self. This robust effect seems to receive support from several lines of empirical work.

There is rich evidence showing that one's name, as well as images of one's face are processed faster and more accurately than other comparable stimuli (Alexopoulos et al. 2012; Alzueta, et al. 2019). This effect seems to be in place early in development, with children between 6 and 10 years of age already showing self-prioritization (Maire et al. 2020). More recently, it has been demonstrated that a similar effect can be reliably observed also for non-familiar stimuli (e.g. geometrical shapes or avatar faces) that have been arbitrarily associated with the self (Sui et al. 2009) 
491 The self prioritization effect has been observed in instances when compared with family and

492 friends' faces (Keyes \& Brady 2010), suggesting that the prioritization is not due to a

493 familiarization effect (e.g. highly learned perceptual information), but rather it is related to the

494 special meaning that the self-face has for one's sense of self. Taken together, these studies

495 are consistent with our findings showing that imagined social touch experiences as received

496 by the self vs the other are rated as being more pleasant in people with low occurrences of

497 depersonalisation experiences.

500 Interestingly however, while it is fairly well established that people benefit from affiliative 501 touch directed to the self (Gallace and Spence 2010), it is less clear why do people with high

502 DP do not show the same self-prioritization effect. Again, there are several potential

503 interpretations of these results.

505 First, one alternative is to argue, in line with Ward \& Banissy (2015) that DP is associated 506 with reduced self-other distinction, which plays a key role in vicarious tactile perception.

507 Within this theoretical framework, atypical representations of bodily self have been linked to 508 individuals who experience vicarious pain. For example, Bowling and colleagues (2019)

509 found a strong positive relationship between trait depersonalisation and the tendency to 510 experience pain on their own body when viewing others in pain (mirror-pain synaesthesia).

511 They proposed that "impairments in the ability to distinguish and switch between self- and 512 other-relevant representations underlie conscious vicarious experience" ( Bowling et al.

513 2019:2). The key hypothesis here is that the self-other distinction is blurred, reduced, which 514 may alter the ability of high DP people to optimally keep track of one's self, and benefit 515 thereby from the self-bias effect.

517 Previous work by Adler and colleagues (2016) used electroencephalography (EEG) to 518 demonstrate that increased DP experiences among healthy adults is associated with altered 519 tactile mirroring mechanisms with less of an activation for self-face related information 520 compared to those with low experience of DP. They argue that mirroring, especially for 521 events on one's own face can be strongly affected by how connected the person feels to their 522 own bodily self. Their findings are in line with several studies suggesting a functional link 523 between self-related processing and mirroring processes (Cardini et al. 2011; Tajadura524 Jimenez et al. 2013; Serino et al. 2015). 
527 This hypothesis received further support from a recent study by Farmer and colleagues (2020) using the Visual Remapping of Touch (VRT; Serino et al., 2008) paradigm to explore selfbias in visual tactile integration in non-clinical participants reporting high and low levels of depersonalisation experiences. In typical population, the VRT effect is enhanced by selfrelevance with as stronger effect for one's own compared to another's face (Serino et al., 2008), suggesting that self-representation plays a key role in mediating this process. A similar effect has also been found for other body parts, namely the hands (Gillmeister, 2014), suggesting that the VRT effect generalises to tactile perception in general. Strikingly, Farmer and colleagues found that the high DP group showed a greater VRT effect when observing the face of the other as opposed to the self.

A second route for interpreting why high DP people do not benefit from the self-prioritization effect because of the potential top-down conflicts between explicit self-recognition and feelings of detachment from the observed self. This hypothesis seems to be supported by phenomenological self-reports of people with depersonalisation who consistently report viewing their own face as if it was that of a stranger: "The loss of the sense of self is a constantly perturbing experience. Looking at my face in the mirror feels like an uncomfortable staring contest with a total stranger" ( Perkins 2021:41). Intriguingly, these sensations of self-estrangement seem to be closely linked with feelings of disembodiment and detachment from the reality: "I look in the mirror and it doesn't feel like myself I'm looking at. It's like I'm floating, not actually experiencing the world, and slowly fading away into nothing. It's like I'm on autopilot in somebody's else body" (Perkins 2021 :198) (see also Simeon and Abugel 2006; Sierra 2009).

A third route to explain why DP people do not show preference for touch experiences received by the self may be related to alterations in sensory imagery. For example, Lambert and colleagues ( 2001) conducted a study on twenty-eight people diagnosed with depersonalisation disorder (DDD) using self-report measures of imagery ability in relation to a range of symptoms and in comparison with age- and sex-matched controls. They found that symptoms of depersonalisation correlated with impaired ability to generate visual images. This was particularly evident with images pertaining to the self and other people as opposed to objects. It is currently an open question whether these alterations may apply also to all 
sensory modalities (e.g. imagined touch versus imagined pictures). Our study seems to suggest that tactile imagery may be impaired as well in people with high DP. Further work is needed to answer these key questions, by contrasting imagery abilities in different sensory modalities in people with DP.

Another notable finding is that high DP individuals rate "get" (being touched) items significantly lower compared to low DP individuals. However, this is not the case for "give" (touching) items. This seems to suggest again a tendency to move away from the sensing self. Additionally, our study reveals a significant main effect for pleasantness on where the touch is delivered, confirming previous reports with the imagined "back" touch scenarios being rated significantly more pleasant than the dorsal forearm in both groups (Walker et al. 2017).

While our first experiment consisted in asking participants to passively watch short videos of CT-optimal stroking and to rate vicarious tactile experiences of affective touch in relation to the self and other, in experiment $\mathrm{n}^{\circ} 2$ we have added a dynamic component. Specifically, our CT-optimal self-touch intervention on one's body (dorsal forearm) yielded several key results. First, as anticipated we found that the dynamic CT-optimal self-touch stroking intervention (as opposed to passive self-touch, i.e. right palm resting on the forearm) enhanced the vividness of touch experiences in both people with high and low depersonalisation. This is a key aspect, because it suggests that affective touch stroking modulates tactile perception not only when it is delivered by others on one's body, but also when it is delivered by oneself on one's own body. To our knowledge, this is the first study providing evidence that affective self-touch influences one's subjective tactile experiences in DP.

Again, there are several potential interpretations of these findings. First, as briefly mentioned in the Introduction section, previous theoretical and empirical work outlined that among the senses, touch has a special status in relation to both the body and the world and others. As Merleau-Ponty famously pointed out, whilst vision "presents us with a spectacle spread out

588 before us at a distance", in perceiving the world through touch, "I cannot forget in this case 589 that it is through my body that I go to the world" (1962:316). By directly mediating the boundary between body and world, the skin inevitably distinguishes yet relates body and world, as the two faces of the same coin. By gaining information about the world via touch, the individual inherently gains information about oneself 'for free'. 
Moreover, the skin is the oldest and widest organ in terms of dimensions and functions (Montagu, 1971; Field, 2001). By providing the organism with the most primitive means to 'meet' and perceive the world, tactile experiences may constitute thereby the most ubiquitous and basic experiential background ( Ciaunica \&Fotopoulou, 2017; Fotopoulou \& Tsakiris 2017; Ciaunica et al. 2021b).

599

Building upon the idea that affective touch is believed to play an important role to the sense of body ownership (Crucianelli et al., 2013; 2018; van Stralen et al., 2014) and self-identity (Panagiotopoulou et al., 2017), one may speculate that affective self-touch may increase the vividness of perceptual experiences overall. Our findings complement previous work suggesting that voluntary active self-touch (as opposed to passive self-touch) increased body ownership over a virtual hand, showing an important role for active self-touch in the formation of the bodily self ( Hara et al. 2015).

Interestingly, our study found no significant effect of the CT-optimal self-stroking on the pleasantness of the experiences across conditions. As mentioned previously, this suggests that perceived vividness and pleasantness of tactile experiences are underpinned by two distinct mechanisms in relation to self-detachment in DP. One may speculate that the pleasantness aspect of affective touch experiences is intrinsically related to a social component. Seminal studies illustrated that we automatically anticipate the sensory effects of self-initiated actions (Wolpert \& Kawato 1998), which explains why people typically cannot tickle themselves (Blakemore et al., 1998). Similarly, we typically prefer to receive a massage from another close person, rather than a self-massage. By contrast, the vividness of self-touch experiences may be related to a functional, rather than social component. For example, for basic survival reasons, one must be able to keep track of one's body and provide immediate soothing in case of harmful events (e.g. humans typically self-stroke instinctively painful body parts after a harmful stimuli). This suggests that self-touch may have a potential soothing effect and decrease the impact of nociceptive stimuli as shown by work on the role of self-touch in somatosensory and body representation disorders after stroke (van Stralen et al. 2011). were unable to control how accurately our participants followed our instructions (e.g. whether 627 they attentively watched the videos, or whether they correctly performed the CT-optimal self628 stroke on their dorsal forearms as instructed). Moreover, further work needs to contrast the 
conditions where the CT-optimal stroking is delivered on one's body by oneself versus a close/trusted other, or the experimenter in people with high and low DP experiences. For example, it would be interesting to examine whether CT-optimal stroking delivered by others (as opposed to self-touch) will increase both the vividness and pleasantness of tactile experience in both groups (low and high DP). Another important limitation is that our study was conducted during the COVID-19 pandemic. The current exceptional context encourages people to avoid as much as possible proximal tactile interactions with others and the surroundings (aiming at limiting the risks of potential infection). Hence the touch-related social behaviours may be disrupted by this atypical context, and it would important to conduct a follow-up study post-pandemic to confirm the same pattern of findings.

\section{Conclusion}

Depersonalisation is a very common dissociative experience that makes people feel detached from their bodily self. We have conducted an online study featuring two experiments looking at the relationship between DP and affective touch and self-touch.

In experiment 1, we explored to what extent DP traits modulate the perceived pleasantness and vividness of tactile experiences as imagined being received by the self and other. Experiment 2 used a newly designed affective self-touch intervention in order to explore the effect of CToptimal self-touch stroking on one's dorsal forearm on the perceived pleasantness and vividness of tactile experiences as being received by the self and other. We found that DP modulates the vicarious rating of touch experiences, such as low DP individuals reported higher perceived pleasantness and vividness rating for touch. By contrast, the high DP cohort rated all touch experiences as significantly less pleasant. No significant interaction effects for vividness ratings of touch experiences across low and high DP.

In addition, our results suggest that people with low DP rate the perceived pleasantness of the imagined social touch experiences as received by the self higher than if received by the other. Intriguingly, in high DP individuals, there is no difference in the perceived pleasantness of affective touch imagined as being received by the self vs the other. Finally, we found that both low and high DP participants, following our tailored CT-optimal affective self-touch 
663 intervention on one's own body, report significantly higher ratings of vividness of tactile 664 perception.

665

666 Our study points to several potential important implications. For example, the finding that

667 CT-optimal self-touch delivered by both people with low and high DP experiences on their 668 own bodies (dorsal forearms) modulates the perceived vividness of tactile experiences,

669 suggests that our approach may be used to design affective self-touch based interventions.

670 These may help isolated people that feel detached from their own self, body and the world, to 671 enhance the vividness of their experiences and potentially increase their sense of "realness" or 672 "presence " in their own bodies and the world (Ciaunica et al. 2021a).

673

674 This is important because tactile perception is pivotal not only in establishing affective bonds

675 with others, but also in constituting our embodied sense of self. Indeed, DP has highly

676 distressing effects on the quality of one's mental and social life, often and strikingly reported 677 as having an opaque "fog" or "veil" interposed between one's self, body and the world

678 (Simeon \& Abugel, 2006; Sierra 2019; Ciaunica \& Charlton, 2018).

679

680 Given that our skin is not just a mere border (separating the self from the others/world), but 681 also a bridge connecting the self to its environment, then one may use proximal skin-to-skin 682 interactions as a handy mean to 'dissolve' this fog, and to 'get back in touch' with the 'lost' 683 self.

684

685

686

Acknowledgements

687

688

The Authors would like to thank Bigna Lenggenhager for helpful comments on earlier drafts 689 of this manuscript.

\section{Funding}

693 This work was supported by a Bial grant n 157/16, and a FCT grant PTDC/FER-FIL/4802/2020 to

694 AC; OD is funded by a Momentum grant from the Volkswagen Foundation (CO-SENSE) and a grant

695 from the NOMIS foundation (DISE). 


\section{References}

Ackerley, I. Carlsson, H. Wester, H. Olausson, H. Backlund Wasling, Touch perceptions across skin sites: differences between sensitivity, direction discrimination and pleasantness, Front. Behav. Neurosci. 8 (2014) 54, http:// dx.doi.org/10.3389/fnbeh.2014.00054.

Adler, J., Schabinger, N., Michal, M., Beutel, M. E., \& Gillmeister, H. (2016). Is that me in the mirror? Depersonalisation modulates tactile mirroring mechanisms. Neuropsychologia, 85: 148-158.

Adler, J., \& Gillmeister, H. (2019). Bodily self-relatedness in vicarious touch is reflected at early cortical processing stages. Psychophysiology, 56(12), e13465. https://doi.org/10.1111/psyp.13465

Ackner, B. (1954). Depersonalization. I. Aetiology and phenomenology. II. Clinical syndromes. Journal of Mental Science, 100, 838-872. https://doi.org/10.1192/bjp.100.421.838

Alais D, Newell FN, Mamassian P (2010) Multisensory processing in review: from physiology to behaviour. See Perceiving 23(1): 3-38.

Alexopoulos, T., Muller, D., Ric, F., \& Marendaz, C. (2012). I, me, mine: Automatic attentional capture by self-related stimuli. European Journal of Social Psychology, 42, 770-779. doi:DOI: 10.1002/ejsp. 1882

Alzueta, E., Melcón, M., Poch, C., \& Capilla, A. (2019). Is your own face more than a highly familiar face? Biological Psychology.

Anwyl-Irvine, A.L., Massonié J., Flitton, A., Kirkham, N.Z., Evershed, J.K. (2019).

Gorilla in our midst: an online behavioural experiment builder. Behavior Research Methods. Doi: https://doi.org/10.3758/s13428-019-01237-x

American Psychiatric Association, DSM-5 Task Force. (2013). Diagnostic and statistical manual of mental disorders: DSM-5 ${ }^{\mathrm{TM}}$ (5th ed.). American Psychiatric Publishing, Inc.. https://doi.org/10.1176/appi.books.9780890425596

Atzil, S., Gao, W., Fradkin, I. et al. Growing a social brain. Nat Hum Behav 2, 624-636 (2018). https://doi.org/10.1038/s41562-018-0384-6

Baker, D., Hunter, E. C. M., Lawrence, E., Medford, N., Patel, M., Senior, C., ... David, A. S. (2003). Depersonalisation disorder: Clinical features of 204 cases. British Journal of Psychiatry, 182: 428-433.

Beßler, R., Bendas, J., Sailer, U., \& Croy, I. (2019). The "Longing for interpersonal touch picture questionnaire": Development of a new measurement for touch perception. 
International Journal of Psychology, 55(3), 446-455. https://doi.org/10.1002/ijop.12616

Blakemore, S., Wolpert, D. M., \& Frith, C. D. (1998). Central cancellation of self-produced tickle sensation. Nature Neuroscience, 1(7), 635-640. https://doi.org/10.1038/2870

Blakemore, S., Wolpert, C. A. D., \& Frith, C. (2000). Why can't you tickle yourself? NeuroReport, 11(11), 11-16.

Blanke, O., \& Metzinger, T. K. (2009). Full-body illusions and minimal phenomenal selfhood. Trends in Cognitive Sciences, 13(1), 7-13.

Boehme, R., Olausson, H. Differenentiating self-touch from social touch. Current Opinion in Behavioral Sciences, 43:27-33, 2021

Bowling, N. C., Botan, V., Santiesteban, I., Ward, J., \& Banissy, M. J. (2019). Atypical bodily self-awareness in vicarious pain responders. Philosophical Transactions of the Royal Society B, 374(1787), 20180361.

Brown, R. J. (2006). Different types of "Dissociation" have different psychological mechanisms. Journal of Trauma \& Dissociation, 7(4), 7-28. https://doi.org/10.1300/j229v07n04_02

Cardini, F., Costantini, M., Galati, G., Romani, G. L., Làdavas, E., \& Serino, A. (2011). Viewing one's own face being touched modulates tactile perception: An fMRI study. Journal of Cognitive Neuroscience, 23(3), 503-513. https://doi.org/10.1162/jocn.2010.21484

Cardini, F., Tajadura-Jiménez, A., Serino, A., \& Tsakiris, M. (2013). It feels like it's me: Interpersonal multisensory stimulation enhances visual remapping of touch from other to self. Journal of Experimental Psychology: Human Perception and Performance, 39(3), 630-637. https://doi.org/10.1037/a0031049

Ciaunica, A. (2016). Basic Forms of Pre-reflective Self-Consciousness: a Developmental Perspective. In Pre-reflective Self-Consciousness: Sartre and Contemporary Philosophy of Mind, eds. S. Miguens, G. Preyer, and C. Morando, 422-438. London: Routledge. Ciaunica, A. (2020). Introduction: The Relational Self: Basic Forms of Self-Awareness. Topoi, 39: 501-507.

Ciaunica, A. \& Fotopoulou, A. (2017). 'The Touched Self: Psychological and Philosophical Perspectives on Proximal Intersubjectivity and the Self'. In Durt C., Fuchs T., and Tewes C. (eds). Embodiment, Enaction, and Culture-Investigating the Constitution of the Shared World. Cambridge MA: MIT Press, p. 173-192.

Ciaunica, A., Charlton, J. (2018). When the self slips: what depersonalization can say about the self - https://aeon.co/essays/what-can-depersonalisation-disorder-say-about-the-self 
Ciaunica, A., \& Crucianelli, L. (2019). Minimal self-awareness: from within a developmental perspective. Journal of Consciousness Studies, 26(3-4), 207-226.

769

770

771

772

773

774

775

776

777

778

779

780

781

782

783

784

785

786

787

Ciaunica, A., Charlton, J. and Farmer, H., 2020. When the Window Cracks: Transparency and the Fractured Self in Depersonalisation. Phenomenology and the Cognitive Sciences, pp.1-19.

Ciaunica A, Roepstorff A, Fotopoulou AK and Petreca B (2021a) Whatever Next and Close to My Self-The Transparent Senses and the "Second Skin": Implications for the Case of Depersonalization. Front. Psychol. 12:613587. doi: 10.3389/fpsyg.2021.613587

Ciaunica, A., Safron A., Delafield-Butt, J. (2021b). Back to square one: the bodily roots of conscious experiences in early life, Neuroscience of Consciousness, Volume 2021, Issue 2, 2021, niab037, https://doi.org/10.1093/nc/niab037

Ciaunica, A., McEllin, L., Kiverstein, J., Gallese, V., Hohwy, J., \& Wozniak, M. (2021,c) September 21). Zoomed out? Depersonalization is Related to Increased Digital Media Use During the COVID-19 Pandemic Lockdown. https://doi.org/10.31234/osf.io/8jver

Crucianelli, L., N. K. Metcalf, A. K. Fotopoulou, and P. M. Jenkinson. 2013. Bodily pleasure matters: velocity of touch modulates body ownership during the rubber hand illusion. Frontiers in Psychology 4: 703.

Crucianelli, L., Krahé, C., Jenkinson, P.M. and Fotopoulou, A.K., 2018. Interoceptive ingredients of body ownership: Affective touch and cardiac awareness in the rubber hand illusion. Cortex, 104, pp.180-192.

Damasio, A., (2000): The Feeling of What Happens. Body, Emotion and the Making of Consciousness, London: Vintage

Delafield-Butt, J.T. and Gangopadhyay, N., 2013. Sensorimotor intentionality: The origins of intentionality in prospective agent action. Developmental Review, 33(4), pp.399-425.

Deroy, O., Chen, Y. C., \& Spence, C. (2014). Multisensory constraints on awareness. Philosophical Transactions of the Royal Society B: Biological Sciences, 369(1641), 20130207.

Deroy, O., \& Fairhurst, M. (2019). Spatial certainty: feeling is the truth. In Spatial Senses (pp. 183-198). Routledge.

Dewe, H., Watson, D.G., Kessler, K., Braithwaite, J.J., 2018. The depersonalized brain: New evidence supporting a distinction between depersonalization and derealization from discrete patterns of autonomic suppression observed in a non-clinical sample. Conscious. Cogn. 63, 29-46.

Fairhurst, M. T., Löken, L., \& Grossmann, T. (2014). Physiological and Behavioral Responses Reveal 9-Month-Old Infants' Sensitivity to Pleasant Touch. Psychological 
Science, 25(5), 1124-1131. https://doi.org/10.1177/0956797614527114

803

804

805

806

807

808

809

810

811

812

813

814

815

816

817

818

819

820

821

822

823

824

825

826

827

828

829

830

831

832

833

834

835

836

Fairhurst, M. T., Travers, E., Hayward, V., \& Deroy, O. (2018). Confidence is higher in touch than in vision in cases of perceptual ambiguity. Scientific reports, 8(1), 1-9

Farmer, H., Cataldo, A., Adel, N., Wignall, E., Gallese, V., Deroy, O., Hamilton, A., \& Ciaunica, A. (2020). The Detached Self: Investigating the Effect of Depersonalisation on Self-Bias in the Visual Remapping of Touch, Multisensory Research, , 1-22. doi: https://doi.org/10.1163/22134808-bja10038

Faivre, N., Arzi, A., Lunghi, C. and Salomon, R., 2017. Consciousness is more than meets the eye: a call for a multisensory study of subjective experience. Neuroscience of consciousness, 2017(1), p.nix003.

Ferrè, E. R., \& Haggard, P. (2016). The vestibular body: Vestibular contributions to bodily representations. Cognitive Neuropsychology, 33(1-2), 67-81.

Field, T., 2001. Touch. Boston: The MIT Press.

Friston, K. 2010. The free-energy principle: a unified brain theory. Nature Reviews Neuroscience, 11: 127-138.

Fotopoulou, A. and Tsakiris, M., 2017. Mentalizing homeostasis: The social origins of interoceptive inference. Neuropsychoanalysis, 19(1), pp.3-28.

Gallace, A., and C. Spence., 2010. The science of interpersonal touch: An overview. Neuroscience and Biobehavioral Reviews 34 (2):246-259.

Gallagher, S. (2000). Philosophical conceptions of the self: implications for cognitive science. Trends in cognitive sciences, 4(1): 14-21.

Gazzola, V., Spezio, M. L., Etzel, J. A., Castelli, F., Adolphs, R., \& Keysers, C. (2012). Primary somatosensory cortex discriminates affective significance in social touch. Proceedings of the National Academy of Sciences of the United States of America, 109(25), E1657-E1666.

Gillmeister, H. (2014). A new perceptual paradigm to investigate the visual remapping of others' tactile sensations onto one's own body shows "mirror touch" for the hands. Frontiers in Psychology, 5(FEB), 1-9. https://doi.org/10.3389/fpsyg.2014.00095

Gillmeister, H., Bowling, N., Rigato, S., \& Banissy, M. J. (2017). Inter-Individual Differences in Vicarious Tactile Perception: a View Across the Lifespan in Typical and Atypical Populations. Multisensory research, 30(6), 485-508. https://doi.org/10.1163/2213480800002543

Gottlieb, G. (1976). Conceptions of prenatal development: Behavioral embryology. Psychological Review, 83(3), 215-234. https://doi.org/10.1037/0033-295X.83.3.215 Guralnik, O., Schmeidler, J., \& Simeon, D. (2000). Feeling unreal: Cognitive processes in 
depersonalization. American Journal of Psychiatry, 157(1): 103-109.

Haggarty, C. J., Trotter, P. D., McGlone, F., \& Walker, S. C. (2021). Children's vicarious ratings of social touch are tuned to the velocity but not the location of a caress. PloS one, 16(8), e0256303. https://doi.org/10.1371/journal.pone.0256303

Hara, M., Pozeg, P., Rognini, G., Higuchi, T., Fukuhara, K., Yamamoto, A., Higuchi, T., Blanke, O., \& Salomon, R. (2015). Voluntary self-touch increases body ownership. Frontiers in psychology, 6, 1509. https://doi.org/10.3389/fpsyg.2015.01509

Hata, T., 2016. Current status of fetal neurodevelopmental assessment: Four-dimensional ultrasound study. Journal of Obstetrics and Gynaecology Research, 42(10), pp.12111221.

Holmes, E., Brown, R., Mansell, W., Fearon, R., Hunter, E., Frasquilho, F., \& Oakley, D. (2005). Are there two qualitatively distinct forms of dissociation? A review and some clinical implications. Clinical Psychology Review, 25(1), 1-23. https:// doi.org/10.1016/j.cpr.2004.08.006

Hunter, E. C. M., Phillips, M. L., Chalder, T. et al (2003) Depersonalisation disorder: a cognitive-behavioural conceptualization. Behaviour Research and Therapy, 41, 14511467

Hunter EC., Sierra M, David AS (2004) .The epidemiology of depersonalization and derealisation. A systematic review. Society of Psychiatry Psychiatric Epidemiology, 39: $9-18$.

Jáuregui Renaud, K. (2015). Vestibular function and depersonalization/derealization symptoms. Multisensory Research, 28(5-6), 637-651. https://doi.org/10.1163/22134808-00002480

Keyes H, Brady N. (2010) Self-face recognition is characterized by "bilateral gain" and by faster, more accurate performance which persists when faces are inverted. Quarterly Journal of Experimental Psychology. 2010;63(5):840-847. doi:10.1080/17470211003611264

Keysers, C., \& Gazzola, V. (2009). Expanding the mirror: Vicarious activity for actions, emotions, and sensations. Current Opinion in Neurobiology, 19(6), 666-671. https://doi.org/10.1016/j.conb.2009.10.006

Kirsch, L.P., Krahé, C., Blom, N., Crucianelli, L., Moro, V., Jenkinson, P.M. and Fotopoulou, A., 2018. Reading the mind in the touch: Neurophysiological specificity in the communication of emotions by touch. Neuropsychologia, 116, pp.136-149. 
870

871

872

873

874

875

876

877

878

879

880

881

882

883

884

885

886

887

888

889

890

891

892

893

894

895

896

897

898

899

900

901

902

903

904

Kirsch, L.P., Besharati, S., Papadaki, C., Crucianelli, L., Bertagnoli, S., Ward, N., Moro, V., Jenkinson, P.M. and Fotopoulou, A., 2020. Damage to the right insula disrupts the perception of affective touch. Elife, 9, p.e47895.

Krahé, C., Drabek, M.M., Paloyelis, Y. and Fotopoulou, A., 2016. Affective touch and attachment style modulate pain: a laser-evoked potentials study. Philosophical Transactions of the Royal Society B: Biological Sciences, 371(1708), p.20160009.

Lambert, M. V., Senior, C., Phillips, M. L., Sierra, M., Hunter, E., \& David, A. S. (2001). Visual imagery and depersonalisation. Psychopathology, 34(5), 259-264. https://doi.org/10.1159/000049319

Lebois, L. A. M., Wolff, J. D., Hill, S. B., Bigony, C. E., Winternitz, S., Ressler, K. J., \& Kaufman, M. L. (2018). Preliminary evidence of a missing self bias in face perception for individuals with dissociative identity disorder. Journal of Trauma \& Dissociation, 00(00), 1-25. https://doi.org/10.1080/15299732.2018.1547807

Lee WE, Kwok CH, Hunter EC, Richards M, David AS (2012) Prevalence and childhood antecedents of depersonalization syndrome in a UK birth cohort. Soc Psychiatry Psychiatr Epidemiol 47: 253-261.

Liljencrantz, J., Strigo, I., Ellingsen, D. M., Krämer, H. H., Lundblad, L. C., Nagi, S. S., Leknes, S., \& Olausson, H. (2017). Slow brushing reduces heat pain in humans. European journal of pain (London, England), 21(7), 1173-1185. https://doi.org/10.1002/ejp.1018

Leiner, D. J. (2019). SoSci Survey (Version 3.1.06) [Computer software]. Available at https://www.soscisurvey.de

Lemche, E., Brammer, M. J., David, A. S., Surguladze, S. A., Phillips, M. L., Sierra, M., ... Giampietro, V. P. (2013). Interoceptive-reflective regions differentiate alexithymia traits in depersonalization disorder. Psychiatry Research - Neuroimaging, 214(1), 6672. https://doi.org/10.1016/j.pscychresns.2013.05.006

Lemche E, Surguladze SA, Brammer MJ, Phillips ML, Sierra M, David AS, Williams SC, Giampietro VP (2016). Dissociable brain correlates for depression, anxiety, dissociation, and somatization in depersonalization-derealization disorder. CNS Spectr. 2016 Feb;21(1):35-42. doi: 10.1017/S1092852913000588.

Lewis, M., Sullivan, M. W., Stanger, C , \& Weiss, M. (1989). Self development and self-conscious emotions. Child Development, 60, 146- 156.

Limanowski, J. and Friston, K., 2018. 'Seeing the dark': Grounding phenomenal transparency and opacity in precision estimation for active inference. Frontiers in psychology, 9 , p.643. 
Löken, L. S., Wessberg, J., Morrison, I., McGlone, F., \& Olausson, H., 2009. Coding of pleasant touch by unmyelinated afferents in humans. Nature Neuroscience, 12, 547-548

Maire, H., Brochard, R., \& Zagar, D. (2020). A Developmental Study of the SelfPrioritization Effect in Children Between 6 and 10 Years of Age. Child development, 91(3), 694-704. https://doi.org/10.1111/cdev.13352

McGlone, F., Å. Vallbo, H. Olausson, L. Löken, and J. Wessberg., 2007. Discriminative touch and emotional touch. Canadian Journal of Experimental Psychology 61 (3):173183.

McGlone, F., Wessberg, J. and Olausson, H., 2014. Discriminative and affective touch: sensing and feeling. Neuron, 82(4), pp.737-755.

Medford, N., 2012. Emotion and the unreal self: depersonalization disorder and deaffectualization. Emotion Review, 4(2), pp.139-144.

Medford, N., Sierra, M., Baker, D., \& David, A. (2005). Understanding and treating depersonalisation disorder. Advances in Psychiatric Treatment, 11(2), 92-100. doi:10.1192/apt.11.2.92

Medford N, Sierra M, Stringaris A, Giampietro V, Brammer MJ, David AS. Emotional Experience and Awareness of Self: Functional MRI Studies of Depersonalization Disorder (2016). Front Psychol. 2016 Jun 2;7:432. doi: 10.3389/fpsyg.2016.00432.

Merleau-Ponty, M. (1945). Phénoménologie de la perception, Paris: Éditions Gallimard; English translation: C. Smith (1962). Phenomenology of Perception. London: Routledge and Kegan Paul.

Michal, M., Reuchlein, B., Adler, J., Reiner, I., Beutel, M.E., Vögele, C., Schächinger, H., Schulz, A., 2014. Striking discrepancy of anomalous body experiences with normal interoceptive accuracy in depersonalization- derealization disorder. PLoS One 9, e89823.

Microsoft Corporation. (2018). Microsoft Excel. Retrieved from https://office.microsoft.

Millman, L., Hunter, E., Orgs, G., David, A. S., \& Terhune, D. B. (2021). Symptom variability in depersonalization-derealization disorder: A latent profile analysis. Journal of clinical psychology, 10.1002/jclp.23241. Advance online publication. https://doi.org/10.1002/jclp.23241

Montagu, A., 1971. Touching: The Human Significance of the Skin. New York: Columbia University Press.

Northoff, G., and Panksepp, J. (2008). The trans-species concept of self and the subcorticalcortical midline system. Trends Cogn. Sci. 12, 259-264. doi: 10.1016/j.tics.2008.04.007 
Nummenmaa, L., Suvilehto, J. T., Glerean, E., Santtila, P., \& Hietanen, J. K. (2016). Topography of Human Erogenous Zones. Archives of sexual behavior, 45(5), 12071216. https://doi.org/10.1007/s10508-016-0745-Z

Owens, A. P., David, A. S., Low, D. A., Mathias, C. J., \& Sierra-Siegert, M. (2015). Abnormal cardiovascular sympathetic and parasympathetic responses to physical and emotional stimuli in depersonalization disorder. Frontiers in Neuroscience, 9, 89. https://doi.org/10.3389/fnins.2015.00089

Panagiotopoulou, E., Filippetti, M.L., Tsakiris, M. and Fotopoulou, A., 2017. Affective touch enhances self-face recognition during multisensory integration. Scientific reports, 7(1), pp.1-10.

Panksepp, J. 1998. Affective Neuroscience: The Foundations of Human and Animal Emotions. Oxford: Oxford University Press.

Pawling, R., Cannon, P. R., McGlone, F. P., \& Walker, S. C. (2017). C-tactile afferent stimulating touch carries a positive affective value. PloS one, 12(3), e0173457. https://doi.org/10.1371/journal.pone.0173457

Pearce, E., Wlodarski, R., Machin, A., \& Dunbar, R. (2017). Variation in the $\beta$-endorphin, oxytocin, and dopamine receptor genes is associated with different dimensions of human sociality. Proceedings of the National Academy of Sciences of the United States of America, 114(20), 5300-5305. https://doi.org/10.1073/pnas.1700712114

Perkins, J. ( 2021). Life on Autopilot - A Guide to Living with Depersonalisation Disorder. Jessica Kingsley Publishers

Ratcliffe, M., 2013. Touch and the sense of reality. The hand: An organ of the mind, pp.131157.

Rochat, P., and Striano, T., 2000. Perceived Self in Infancy. Infant Behavior and Development, Vol. 23, Issue 3-4, pp. 513-530.

RStudio Team (2020). RStudio: Integrated Development for R. RStudio, PBC, Boston, MA URL http://www.rstudio.com/

Qin P, Wang M, Northoff G. Linking bodily, environmental and mental states in the self-A three-level model based on a meta-analysis. Neuroscience and Biobehavioral Reviews. 2020 May;115:77-95. DOI: 10.1016/j.neubiorev.2020.05.004.

Schabinger N, Gillmeister H, Berti S, Michal M, Beutel ME, Adler J. Detached and distracted: ERP correlates of altered attentional function in depersonalisation. Biol Psychol. 2018 Apr;134:64-71. doi: 10.1016/j.biopsycho.2018.02.014.

Schulz A, Köster S, Beutel ME, Schächinger H, Vögele C, Rost S, Rauh M, Michal M. (2015). Altered patterns of heartbeat-evoked potentials in 

depersonalization/derealization disorder: neurophysiological evidence for impaired cortical representation of bodily signals. Psychosom Med. 2015 Jun;77(5):506-16. doi: 10.1097.

Serino, A., Giovagnoli, G., \& Làdavas, E. (2009). I feel what you feel if you are similar to me. PloS one, 4(3), e4930.

Serino, A., Pizzoferrato, F., \& Làdavas, E. (2008). Viewing a face (especially one's own face) being touched enhances tactile perception on the face. Psychological Science, 19(5), 434-438. https://doi.org/10.1111/j.1467-9280.2008.02105.x

Seth, A. K., \& Tsakiris, M. (2018). Being a beast machine: The somatic basis of selfhood. Trends in Cognitive Sciences, 22(11): 969-981.

Sierra M. (2009). Depersonalisation: A New Look at a Neglected Syndrome. Cambridge: Cambridge University Press.

Sierra M. \& Berrios GE. (1998). Depersonalization: neurobiological perspectives. Biological Psychiatry, 44: 898-908.

Sierra, M., \& Berrios, G. E. (2000). The Cambridge Despersonalization Scale: A new instrument for the measurement of despersonalization. Psychiatry Research, 93: 153164.

Sierra, M., Senior, C., Dalton, J., McDonough, M., Bond, A., Phillips, M. L., ... David, A. S. (2002). Autonomic response in depersonalization disorder. Archives of General Psychiatry, 59(9), 833-838. https://doi.org/10.1001/archpsyc.59.9.833

Sierra, M., \& David, A. S. (2011). Depersonalization: A selective impairment of selfawareness. Consciousness and Cognition, 20(1): 99-108

Sierra, M., Baker, D., Medford, N., \& David, A. S. (2005). Unpacking the depersonalization syndrome: An exploratory factor analysis on the Cambridge Depersonalization Scale. Psychological Medicine, 35(10): 1523-1532.

Simeon, D., \& Abugel, J. (2006). Feeling Unreal: Depersonalization Disorder and the Loss of the Self. Oxford: Oxford University Press.

Simeon, D., Guralnik, O., Hazlett, E. A., Spiegel-Cohen, J., Hollander, E., \& Buchsbaum, M. S. (2000). Feeling unreal: A PET study of depersonalization disorder. American Journal of Psychiatry, 157(11): 1782-1788.

Simeon, D., Knutelska, M., Nelson, D., \& Guralnik, O. (2003). Feeling Unreal: A Depersonalization Disorder Update of 117 Cases. The Journal of Clinical Psychiatry, 64(9): 990-997. 
Sui, J., Liu, C. H., Wang, L., \& Han, S. (2009). Attentional orientation induced by temporarily established self-referential cues. Q J Exp Psychol (Hove), 62(5), 844-849. doi:10.1080/17470210802559393

Tibubos, A.N., Grammes, J., Beutel, M.E., Michal, M., Schmutzer, G., Brähler, E., 2018. Emotion regulation strategies moderate the relationship of fatigue with depersonalization and derealization symptoms. J. Affect. Disord. 227, 571-579.

Tsakiris M. (2017) The multisensory basis of the self: From body to identity to others. Quarterly Journal of Experimental Psychology. 2017;70(4):597-609.

Tajadura-Jimenez, A., Lorusso, L., \& Tsakiris, M. (2013). Active and passive touch during interpersonal multisensory stimulation change self-other boundaries. Consciousness and Cognition, 22(4), 1352-1360.

van Heugten-van der Kloet, D., Giesbrecht, T., Merckelbach, H., 2015. Sleep loss increases dissociation and affects memory for emotional stimuli. J. Behav. Ther. Exp. Psychiatry 47, 9-17.

von Mohr, M., Kirsch, L.P. and Fotopoulou, A., 2017. The soothing function of touch: affective touch reduces feelings of social exclusion. Scientific reports, 7(1), pp.1-9.

von Mohr, M., Krahé, C., Beck, B. and Fotopoulou, A., 2018. The social buffering of pain by affective touch: a laser-evoked potential study in romantic couples. Social cognitive and affective neuroscience, 13(11), pp.1121-1130.

van Stralen, H. E., van Zandvoort, M. J., Hoppenbrouwers, S. S., Vissers, L. M., Kappelle, L. J., \& Dijkerman, H. C. 2014. Affective touch modulates the rubber hand illusion. Cognition, 131(1), 147-158.

van Stralen H. E., van Zandvoort M. J. E. and Dijkerman H. C. 2011The role of self-touch in somatosensory and body representation disorders after stroke Phil. Trans. R. Soc. B3663142-3152 http://doi.org/10.1098/rstb.2011.0163

Walker, S. C., Trotter, P. D., Woods, A., \& McGlone, F. (2017). Vicarious ratings of social touch reflect the anatomical distribution \& velocity tuning of C-tactile afferents: A hedonic homunculus?. Behavioural brain research, 320, 91-96. https://doi.org/10.1016/j.bbr.2016.11.046

Ward, J., \& Banissy, M. J. (2015). Explaining mirror-touch synesthesia. Cognitive neuroscience, 6(2-3), 118-133. https://doi.org/10.1080/17588928.2015.1042444

Wilhelm, F. H., Kochar, A. S., Roth, W. T., \& Gross, J. J. (2001). Social anxiety and response to touch: incongruence between self-evaluative and physiological reactions. Biological psychology, 58(3), 181-202.

Zahavi, D. (2005). Subjectivity and selfhood: Investigating the first-person perspective. 
1042 Cambridge, MA: MIT Press.

1043

1044

1045

1046

1047

1048

1049

1050

1051

1052

1053 